\title{
Complicated acute appendicitis presenting as a necrotizing fasciitis of the abdominal wall: a case report
}

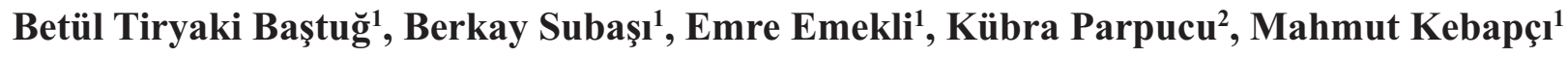 \\ ${ }^{1}$ Department of Radiology, Eskişehir Osmangazi University School of Medicine, Eskişehir, Turkey \\ ${ }^{2}$ Department of Emergency Medicine, Eskişehir Osmangazi University School of Medicine, Eskişehir, Turkey
}

DOI: $10.18621 /$ eurj.346464

\begin{abstract}
Acute appendicitis is one of the most common surgical diseases, but necrotizing fasciitis of the abdominal wall because of perforation is extremely rare. A 50-year-old male presented to the emergency department with severe right-sided abdominal pain for a week. He was hypothermic, hypotensive, and tachycardic. His abdomen was distended, with a large, tender, erythematous region over the right abdominal wall. Laboratory evaluation revealed leukocytosis, acute kidney injury. Computed tomography revealed large collections of fluid and gas in the right abdominal wall as well as inflammation surrounding the right colon. The patient was resuscitated with intra-venous fluid, started on broad-spectrum antibiotics, and emergently brought to the operating room. The patient underwent an exploratory laparotomy, and was found to have appendicitis, which perforated into his abdominal wall resulting in a necrotizing soft tissue infection. It is important to recognize this complication early and proceed immediately to the operating room.
\end{abstract}

Keywords: Perforated appendicitis, abdominal wall abscess, necrotizing fasciitis

Received: October 25, 2017; Accepted: December 11, 2017; Published Online: February 27, 2018

\begin{abstract}
A cute appendicitis is one of the most common surgical diseases, but necrotizing fasciitis of the abdominal wall because of perforation is extremely rare. The diagnosis of perforated appendicitis resulting in necrotizing fasciitis is often delayed due to the unusual presentation of this common disease. Necrotizing fasciitis is associated with significant mortality and requires immediate intervention.

We present a case with complicated acute appendicitis, which perforated into abdominal wall resulting in a necrotizing soft tissue infection.
\end{abstract}

\section{CASE PRESENTATION}

A 50-year-old man presented to our emergency department with abdominal pain for a week. Over the preceding 3 days, the patient's condition had declined rapidly with general weakness and severe escalating but rather superficial abdominal pain. He denied additional symptoms, including nausea, emesis, constipation and diarrhea. He had no history of similar episodes in the past.

On admission, the patient complained of abdominal pain. His temperature was $35.1^{\circ} \mathrm{C}$, blood pressure was $80 / 50 \mathrm{mmHg}$, and heart rate was 90 beats/minute. His physical examination revealed an irregular patch of erythema and tenderness in the right lower quadrant, and crepitation on palpation. The skin in the area of the erythema was hypoesthesic.

Initial laboratory test results showed a C-reactive protein of $330 \mathrm{mg} / \mathrm{L}$ and revealed leukocytosis (white

Address for correspondence: Betül Tiryaki Baştuğ, MD., Assistant Professor, Eskişehir Osmangazi University School of Medicine, Department of Radiology, Eskişehir, Turkey.

E-mail: ,betultryak@yahoo.com 
blood cell count of 14.4 ) with $81 \%$ neutrophils. His serum creatinine was $0.54 \mathrm{mg} / \mathrm{dL}$, sodium was 132 $\mathrm{mmol} / \mathrm{L}$, potassium was $5.47 \mathrm{mmol} / \mathrm{L}$ and glukoz was $330 \mathrm{mg} / \mathrm{dL}$. Hemoglobin level was $9.6 \mathrm{gr} / \mathrm{dL}$. An immediate abdominal computed tomography (CT) was performed. CT scan revealed collections of fluid and gas in the right inferoposterior abdominal wall as well as inflammation surrounding the right colon (Figure 1). The patient was resuscitated with intravenous fluid, started on broad-spectrum antibiotics, and emergently brought to the operating room. There was no intraabdominal free fluid. However subcutaneous collection was noted.

The patient underwent an exploratory laparotomy, and was found to have appendicitis, which perforated into his abdominal wall resulting in a necrotizing soft tissue infection. He underwent a washout, and abdominal wall debridement. Cultures grew Enterococcus faecium, Escherichia coli and Enterobacter aerogenes. Pathology of the skin and subcutaneous tissue was consistent with acute inflammation and necrosis.

\section{DISCUSSION}

Necrotizing fasciitis is a rare soft tissue infection. Disease can involve the superficial fascia, subcutaneous fat, and deep fascia. Giuliano et al. [1] divided necrotizing fasciitis into two groups, based on the analysis of bacteriologic culture results: Type 1, which is polymicrobial and involves non-group A streptococci plus anaerobes and/or facultative anaerobes and also often involves enterobacteriaceae; Type 2, also known as hemolytic streptococcal gangrene, in which the pathogen population is composed of group A $\beta$-hemolytic streptococci alone or in combination with a Staphylococcus bacterium.

Necrotizing fasciitis can occur in any region of the body, but is found most commonly in the abdominal wall, extremities, and perineum [2].

A diagnosis of necrotizing fasciitis is primarily based on the clinical and physical examinations. Patients complain of severe pain. The examination features are edema, tenderness, cutaneous erythema, crepitus, and skin vesicles. CT examination is very useful for confirming the diagnosis of necrotizing fasciitis.

Perforated appendicitis resulting in necrotizing fasciitis of the abdominal wall is rare. This occurs when the appendix perforates into abdominal wall tissues and causes a rapidly progressive bacterial infection of the fascia. The diagnosis is often delayed due to the unusual presentation. Necrotizing fasciitis is a life-threatening event, which requires urgent debridement and broad-spectrum antibiotic treatment, as delay in debridement is associated with significant mortality [3].
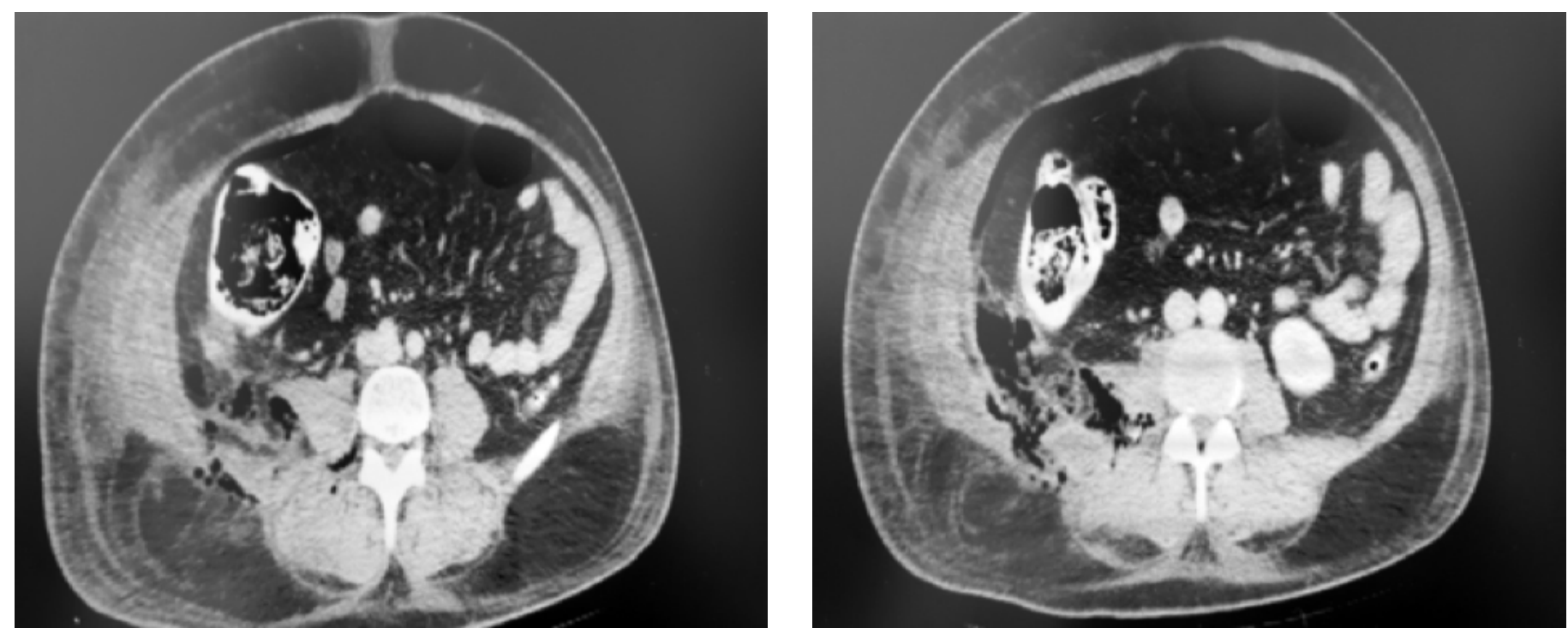

Figure 1. Axial images from a computed tomography scan of the abdomen revealing collections of fluid and gas in the right inferoposterior abdominal wall as well as inflammatory change adjacent to the right colon 
Necrotizing fasciitis due to a perforated appendix is nearly always associated with perforation of a retrocecal appendix due to delayed diagnosis and treatment. There are two orifices that have been described: the inferior and superior lumbar triangles (known as Petit and Grynfeltt-Lesshaft triangles). As these two triangles void of muscular layers are areas of relative weakness in the abdominal wall [4]. Retroperitoneal inflammation resulting from perforated acute appendicitis can pass through these two triangles to the flank and the lumbar area, and can lead to necrotizing fasciitis [5].

Selected axial views of CT from our case showing retroperitoneal inflammation and abscesses resulting from perforated acute appendicitis that passes through these two triangles to the flank and the lumbar area, leading to the occurrence of necrotizing fasciitis.

Necrotizing soft tissue infections may spread rapidly and develop along fascial planes, skin and underlying muscle [6]. These patients experience pain, fever, rapid deterioration, gas or crepitus, and a systemic inflammatory response syndrome with elevated inflammatory markers [7].

Our patient showed rapidly progressing erythema with severe pain, hemodynamic instability, oliguria, crepitation, and septic shock syndrome, which were highly doubtful for necrotizing fasciitis. A Risk Indicator for Necrotizing Fasciitis (LRINEC) score developed to distinguish between soft tissue infection and necrotizing fasciitis was 8 points, representing a risk of over $90 \%$ for necrotizing fasciitis. This score includes routine laboratory values, such as C-reactive protein, white blood cell count, sodium, creatinine, glucose, and hemoglobin levels [8].

The use of CT is helpful to affirm the diagnosis of a soft tissue infection and it can supply information about involvement of intraabdominal organs. Magnetic resonance imaging (MRI) has been shown to be the most definitive imaging method to differentiate between a simple subcutaneous infection and necrotizing fasciitis [9]. However, MRI examination is time-consuming and not always available. Confirmation or exclusion of the diagnosis of necrotizing fasciitis can be reached only during surgical exploration with histological and microbiological workup.

\section{CONCLUSION}

In Intraabdominal causes (e.g., appendicitis) should be considered when faced with a patient with abdominal wall cellulitis, and cross-sectional abdominal imaging should be performed prior to surgical debridement.

\section{Informed consent}

Written informed consent was obtained from the patient for the publication of this case report.

\section{Conflict of interest}

The authors declared that there are no potential conflicts of interest with respect to the research, authorship, and/or publication of this article.

\section{REFERENCES}

[1] Giuliano A, Lewis F Jr, Hadley K, Blaisdell FW. Bacteriology of necrotizing fasciitis. Am J Surg 1977;134:52-7.

[2] Elliott DC, Kufera JA, Myers RA. Necrotizing soft tissue infections. Risk factors for mortality and strategies for management. Ann Surg 1996;224:672-83

[3] Chen CW, Hsiao CW, Wu CC, Jao SW, Lee TY, Kang JC. Necrotizing fasciitis due to acute perforated appendicitis: case report. J Emerg Med 2010;39:178-80.

[4] Ishigami K, Khanna G, Samuel I, Dahmoush L, Sato Y. Gas-forming abdominal wall abscess: unusual manifestation of perforated retroperitoneal appendicitis extending through the superior lumbar triangle. Emerg Radiol 2004;10:207-9.

[5] Hua J, Yao L, He Z-G, Xu B, Song Z-S. Necrotizing fasciitis caused by perforated appendicitis: a case report. Int J Clin Exp Pathol 2015;8:3334-8.

[6] Gujral S, Hughes JM, Wiberg A. Necrotizing fasciitis. Eplasty 2014;14:ic15.

[7] McHenry CR, Piotrowski JJ, Petrinic D, Malangoni MA. Determinants of mortality for necrotizing soft-tissue infections. Ann Surg 1995;221:558-65.

[8] Wong CH, Khin LW, Heng KS, Tan KC, Low CO. The LRINEC (Laboratory Risk Indicator for Necrotizing Fasciitis) score: a tool for distinguishing necrotizing fasciitis from other soft tissue infections. Crit Care Med 2004;32:1535-41.

[9] Schmid MR, Kossmann T, Duewell S. Differentiation of necrotizing fasciitis and cellulitis using MR imaging. AJR Am J Roentgenol 1998;170:615-20. 